\title{
Multilevel solution of the elastohydrodynamically lubricated circular contact problem Part I: theory and numerical algorithm
}

\author{
C. H. Venner and W. E. ten Napel \\ Faculty of Mechanical Engineering, University of Twente, PO Box 217, 7500AE Enschede \\ (Netherlands)
}

(Received May 1, 1991; accepted July 29, 1991)

\begin{abstract}
This paper describes a fast numerical solver for the elastohydrodynamically lubricated circular contact problem. As a result of the application of multigrid techniques the algorithm is of very low complexity, i.e. $O(n \ln n)$, where $n$ is the number of nodes on the grid. Consequently, it enables the solution of the problem using large numbers of nodes $\left(O\left(10^{5}\right)\right)$ on a small-capacity computer, as is demonstrated by the results presented for an example load situation.
\end{abstract}

\section{Introduction}

In the past few decades a large number of papers has been published dealing with the numerical calculation of the pressure profile and film shape in elastohydrom dynamically lubricated (EHL) contacts. In spite of the fact that it is a less realistic configuration, the majority of the papers has dealt with the (one-dimensional) line contact problem. The main reason that the (two-dimensional) point contact problem is relatively scarcely addressed is most probably its computational complexity.

Firstly, there is a computing time problem. The governing equations are discretized on a grid covering the fluid flow domain. Compared with the line contact problem there is an additional dimension in the equations. As a result, the number of gridpoints needed to describe the pressure profile and film shape in a point contact with the same accuracy that is nowadays quite common for a linc contact is some two or three orders of magnitude larger. For example, if $n$ denotes the number of nodes on the grid, typically $n \geq 10^{4}$ is desirable and even larger $n$ is needed if a local feature, e.g. surface roughness, is to be described too. For various reasons such a large number of nodes causes excessive computing times, even when supercomputers are used.

For example, assume that a local relaxation scheme, e.g. a simple Gauss-Seidel relaxation, is used to solve the discrete system of equations (see e.g. refs 1-4). Advantages of such a relaxation process are the small computer storage requirements and the easy implementation of the cavitation condition. However, to obtain a converged solution requires roughly $O(n)$ relaxation sweeps. For each sweep the elastic deformation of the surfaces must be calculated or updated.

To compute the elastic deformation in each gridpoint requires an integration over the entire domain. Hence, to calculate the elastic deformation in all points requires $O\left(n^{2}\right)$ operations. Consequently, the complexity of such a solver for the EHL problem 
is roughly $O\left(n^{3}\right)$. With the aforementioned number of nodes it is obvious that anmintin time problems can be expected.

The situation for a "global" type of iterative solver such as the so-called vewton-Raphson system approach for example applied by $\mathrm{Oh}$ [5] and $\mathrm{Oh}$ and Rohde $[6]$ is even worse. In that case the necessary number of iterations may he smaller thet the computational cost of one iteration is much larger. Due to the elastic deformation integrals, each iteration requires the inversion of a full $n \times n$ Jacobian matrix, $x^{2}$ operations. Hence, again excessive computing times for large $n$. Besides, a huge ammontua of computer storage is needed for all $O\left(n^{2}\right)$ elements of the Jacobian matrix

The second reason for the computational complexity of the point contact problem arises from stability considerations. The most straightforward numerical solution in gorithms are not stable for the highly loaded conditions that are quite commen in concentrated contacts between steel surfaces, i.e. with a maximum pressure fanging up to some $3.0 \mathrm{GPa}$.

Since the carly 1980 s considerable progress with respect to both the computational cost and the stability problems has been reported. For example, Lubrecht $[7,8]$ introduced multigrid (multilevel) techniques, to deal efficiently with the slowness of convergence of a local relaxation scheme. In principle these techniques enable the solution of the problem up to the level of the discretization error in an amount of work equivalent to roughly $10-20$ relaxation sweeps. Compared with the aforementioned $O(n)$ iterations this gives a significant reduction of the computational cost. The application of these techniques enabled Lubrecht to solve the circular contact problem using a relatively large number of nodes on a small-capacity computer and to carry wut an extensive parametric study. Nevertheless, the complexity of Lubrecht's algorithm was still relatively high, ite. $O\left(n^{2}\right)$, due to the evaluation of the elastic deformation integrals. Furthermore, the simple Gauss-Seidel relaxation on which the algorithm was based gradually becomes unstable with increasing load and Lubrecht could only whin solutions for relatively moderately loaded situations.

With respect to the stability problems significant progress was reported by Kwch et al. [9]. They developed an algorithm for the numerical solution of point contact problems based on the so-called inverse solution techniques. With this algorithm they were able to solve the problem even for the highly loaded situations mentioned above. A similar algorithm was presented by Seabra and Berthe [10]. However, this algorithm is not really suited for large $n$ since its complexity is also at least $O\left(n^{2}\right)$ due to the elastic deformation integrals.

This paper describes the development of an advanced solver for EHL point contact problems along the lines set out by Lubrecht, i.e. based on multigrid techniques. Two important steps have been taken. The first step is that an alternative relaxation scheme has been developed providing stability also for highly loaded conditions. The second step is the implementation of a newly developed multilevel technique called Multilevel Multi-integration. This technique was developed by Brandt and Lubrecht [11] and reduces the computing time needed for the calculation of the type of integrals such as those describing the elastic deformation from $O\left(n^{2}\right)$ to $O(n \ln n)$.

The final result of these two steps is an algorithm solving the steady-state circular contact problem in $O(n \ln n)$ operations also for highly loaded conditions. This low complexity allows solution of the problem using a large number of nodes $(n=250000)$ on a small-capacity computer. For the usual smooth surface situation this number of nodes may seem unnecessarily large, but it demonstrates the prospect of the algorithm for future studies of more complex (transient, surface features, roughness) point contact problems. 


\section{Equations}

Substitution of the following dimensionless variables:

$\ddot{\rho}=\rho / \rho_{0}$

$\ddot{\eta}=\eta / \eta_{0}$

$X=x / a$

$Y=y / a$

$P=p / p_{\mathrm{h}}$

$H=h R / a^{2}$

where $a$ is the radius of the Hertzian contact circle:

$a^{3}=\frac{3 F R_{x}}{2 E^{t}}$

and $p_{\mathrm{h}}$ is the maximum Hertzian pressure:

$p_{\mathrm{h}}=\frac{3 F}{2 \pi a^{2}}$

results in the following dimensionless Reynolds equation for the steady-state twodimensional circular contact problem:

$\frac{\partial}{\partial X}\left(\epsilon \frac{\partial P}{\partial X}\right)+\frac{\partial}{\partial Y}\left(\epsilon \frac{\partial P}{\partial Y}\right)-\frac{\partial(\bar{\rho} H)}{\partial X}=0$

In addition the cavitation condition $P \geq 0$ should be satisfied in the domain and $P=0$ at the boundaries. In this equation $\epsilon$ denotes

$\epsilon=\frac{\bar{\rho} H^{3}}{\bar{\eta} \lambda}$

The dimensionless parameter $\lambda$ is defined according to

$\lambda=\frac{6 \eta_{0} u_{\mathrm{s}} R_{x}^{2}}{a^{3} p_{\mathrm{h}}}$

The dimensionless lubricant density $\ddot{\rho}$ is assumed to depend on the pressure according to the Dowson and Higginson relation [12]. Besides, the Roelands viscosity pressure relation [13] is used.

Substitution of the same dimensionless parameters in the film thickness equation gives

$H(X, Y)=H_{00}+\frac{X^{2}}{2}+\frac{Y^{2}}{2}+\frac{2}{\pi^{2}} \int_{-\infty}^{\infty} \int_{-\infty}^{\infty} \frac{P\left(X^{\prime}, Y^{\prime}\right) \mathrm{d} X^{\prime} \mathrm{d} Y^{\prime}}{\left[\left(X-X^{\prime}\right)^{2}+\left(Y-Y^{\prime}\right)^{2}\right]^{1 / 2}}$

where $H_{00}$ is a constant.

Finally the dimensionless force balance equation, demanding that the integral over the pressure equals the externally applied contact load, reads

$\int_{-\infty}^{\infty} \int_{-\infty}^{\infty} P(X, Y) \mathrm{d} X \mathrm{~d} Y-\frac{2 \pi}{3}=0$ 


\section{Numerical solution}

This section briefly outlines the step-by-step approach leading to a fast solver fot the point contact problem. For a more detailed explanation see ref. 14

\subsection{Discretization}

The equations are discretized on a rectangular uniform grid extended over the domain $\left\{(X, Y) \in \mathbb{R}^{2} \mid X_{a} \leq X \leq X_{b},-Y_{a} \leq Y \leq Y_{a}\right\}$ with mesh size $h$. Using a second-order accurate central discretization for the first two terms of Eqn. (1) and a first-order upstream discretization of the wedge term leads to the following equation to be satisfied at each non-boundary site $(i, j),\left(X_{a}+i h,-Y_{a}+j h\right)$ :

$$
\begin{aligned}
& h^{-2}\left(\epsilon_{i-1 / 2, j}\left(P_{i-1, j}-P_{i, j}\right)+\epsilon_{i+1 / 2, j}\left(P_{i+1, j}-P_{i, j}\right)+\epsilon_{i, j-1 / 2}\left(P_{i, j-1}-P_{i, j}\right)\right. \\
& \left.\quad+\epsilon_{i, j+1 / 2}\left(P_{i, j+1}-P_{i, j}\right)\right)-h^{-1}\left(\bar{\rho}_{i} H_{i}-\bar{\rho}_{i-1} H_{i-1}\right)=0
\end{aligned}
$$

with the cavitation condition: $P_{i, j} \geq 0$.

$\epsilon_{i-1 / 2, j}, \epsilon_{i+1 / 2, j}, \epsilon_{i, j-1 / 2}$ and $\epsilon_{i, j+1 / 2}$ denote the value of $\epsilon$ at the intermediate locations:

$(X, Y)=\left\{\begin{array}{l}\left(X_{a}+\left(i-\frac{1}{2}\right) h,-Y_{a}+j h\right) \\ \left(X_{a}+\left(i+\frac{1}{2}\right) h,-Y_{a}+j h\right) \\ \left(X_{a}+i h,-Y_{a}+\left(j-\frac{1}{2}\right) h\right) \\ \left(X_{a}+i h,-Y_{a}+\left(j+\frac{1}{2}\right) h\right)\end{array}\right.$

respectively.

They are computed in an analogous way as described in ref. 15 for the line contact problem, e.g.

$\epsilon_{i-1 / 2, j} \equiv\left(\epsilon_{i, j}+\epsilon_{i-1, j}\right) / 2$

$\epsilon_{i, j}$ is defined as

$\epsilon_{i, j}=\frac{\bar{\rho}\left(P_{i, j}\right) H_{i, j}^{3}}{\bar{\eta}\left(P_{i, j}\right) \lambda}$

Discretizing the elastic deformation integral for example described by Lubrecht $[7,8]$ gives

$H_{i, j}=H_{00}+\frac{X_{i}^{2}}{2}+\frac{Y_{j}^{2}}{2}+\frac{2}{\pi^{2}} \sum_{k=0}^{n_{k}} \sum_{l=0}^{n_{y}} K_{i k j l}^{h h h h} P_{k l}$

where the coefficients $K_{i k j l}^{\text {hhhh }}$ are determined analytically assuming the pressure to be a piecewise constant function, see eg. refs. 7, 14. Furthermore, $\left(n_{x}+1\right)$ and $\left(n_{y}+1\right)$ denote the number of nodes on the grid in the $X$ and $Y$ directions respectively.

Finally the force balance equation determining the value of the integration constant $H_{00}$ is discretized according to

$h^{2} \sum_{i=1}^{n_{x}-1} \sum_{j=1}^{n_{k}-1} P_{i, j}-\frac{2 \pi}{3}=0$

\subsection{Multilevel techniques}

Equations (4)-(7) will be solved simultaneously using the "standard" multigrid techniques. For an introduction to these techniques the reader is referred to Briggs [16]. The reader is also referred to Brandt [17]. The concept of multilevel (multigrid) 
fast solvers is based upon an understanding of the convergence behaviour of a local relaxation process. In many situations such a process is very efficient in reducing error components with a wavelength of the order of the mesh size, i.e. high-frequency error components. Error components, with wavelengths that are large compared with the mesh size, are hardly effected however. Consequently, after the first few relaxations the error on the grid is smooth compared with the mesh size and, because of the slow reduction of these remaining smooth error components, many relaxation sweeps are needed $(O(n))$ to finally reach a converged solution.

The important step leading to a multilevel solver is that such a smooth error can be accurately represented and solved on a coarser grid. Therefore, instead of continuing the relaxation process when, after a few relaxations, convergence slows down, one switches over to a coarser grid. On that grid an approximation to the smooth error is solved and subsequently this approximation is used to correct the solution on the finer grid. The equations from which the error on the coarse grid must be solved are basically the same as the original problem. Hence, the same iterative procedure can be used. However, compared with the computational cost of iterations on the fine grid, the amount of work needed for the solution of the error on the coarse grid is much smaller. The first reason for this reduction is that the number of nodes on the coarse grid is much smaller and consequently one iteration on this grid requires fewer operations. The second reason is that, because of the larger ratio between the wavelength of the error component and the mesh size, the iterative process on the coarse grid converges faster and a given error reduction requires fewer iterations.

The same reasoning applies to the iterative solution of the error on the coarse grid. If the number of nodes on this grid is relatively large the convergence will slow down again after a few iterations. The remaining error on this grid will be smooth and can be accurately approximated and solved on an even coarser grid. This process can be repeated until a grid is reached on which the problem can be solved in only a few iterations. The result is used to correct the solution of the problem on the next finer grid and so on until the finest grid is reached again. Hence, to solve the problem on some grids, a set of coarser grids is used. On each grid only a few iterations are carried out and only on the coarsest grid is the problem really solved. Usually the number of nodes on the coarsest grid is small so this requires only a few iterations. This sequence of going from the finest grid to the coarsest and back again is generally denoted as a coarse grid correction cycle, see e.g. refs. 16 and 17 . The error reduction that can be obtained in such a cycle is independent of the number of nodes on the finest grid and, since all smooth error components are solved on the coarser grids, it is determined by the efficiency of the relaxation process in reducing the high-frequency error components.

With these techniques the problem can be solved up to the level of the discretization error in a total amount of work that is the equivalent of roughly 10-20 relaxation sweeps on the finest grid whereas otherwise $O(n)$ relaxation sweeps would be needed.

For further details the reader is referred to the aforementioned publications. Furthermore, details with respect to the application to EHL problems can be found in refs. 8 and 15 . In this paper only the relaxation process, the actual basis of the algorithm, is discussed in detail.

In the course of iteratively solving eqns. (4)-(7), the clastic deformation must be calculated, recalculated or updated, for example after each relaxation. This requires carrying out the multi-summation in eqn. (6) which, as explained before, is a very time-consuming task. For that purpose the newly developed algorithm Multilevel Multiintegration will be used. This technique enables evaluation in $O(n \ln n)$ operations. 
It can be easily combined with the above-described multilevel solution techniques. for details see refs. 11,18 and also ref. 15 .

\subsection{Relaxation}

In the preceding section the role of relaxation in a multilevel solver has been explained. From this description it is clear that a multilevel solver for the point contact problem requires a relaxation scheme that efficiently reduces high-frequency error components. In fact, the efficiency of the scheme to reduce error components with a wavelength of the order of magnitude of the mesh size determines the efficiency of the solver. With respect to low-frequency components the only requirement that the relaxation should meet is that they are not amplified, otherwise the process is unstable. As long as these components are not amplified by the relaxation the specific amplitude reduction factor is not very important since they are solved on the coarser grids. The relaxation should meet both requirements, ie. good error smoothing and stability for all conditions, including high loads.

The problems encountered in developing such a scheme for the point contact problem are very similar to the problems one had to overcome in the case of the linc contact problem, see eg. ref. 15 . The coefficient $\epsilon$ in eqn. (1) varies by several orders of magnitude over the calculational domain. Globally in the Hertzian dry contact region $\left(\left(X^{2}+Y^{2}\right) \leq 1\right)$ the viscosity $\bar{\eta}$ is large and $H$ is small. As a result $\epsilon \ll 1$, particularly for high loads. Consequently, in this region eqn. (1) reduces to

$\frac{\partial(\rho H)}{\partial H} \simeq 0$

with $H$ given by eqn. (2). Consequently, the integral aspect of the problem dominates. In the outer part of the domain $\left(\left(X^{2}+Y^{2}\right) \geq 1\right) H$ is large and $\tilde{\eta}=1$. Hence, in this region $\epsilon \gg 1$. As a result the first two terms in eqn. (1) dominate and the problem basically behaves as a differential problem. Furthermore, with increasing load the intermediate region, the boundary layer, narrows with increasing load.

The relaxation process used in a multilevel solver of the problem should be a good error smoother for both large and small values of $\epsilon$. The approach to obtain such a scheme is basically the same as described in ref. 15 for the line contact problem. First a linear model problem is studied. This model problem is chosen in such a way that it is characteristic for the local behaviour of the full problem. Subsequently, using the results of the analysis of this linear problem, step by step, a relaxation process for the full problem has been developed.

\subsubsection{Linearized problem}

The following linear approximation of the EHL circular contact problem, i.e. eqn. (1), is studied:

$L(P)=\epsilon\left(\frac{\partial^{2} P}{\partial X^{2}}+\frac{\partial^{2} P}{\partial Y^{2}}\right)-\frac{\partial H}{\partial X}=0$

on the domain $\left\{(X, Y) \in \mathbb{R}^{2} \mid X_{a} \leq X \leq X_{b},-Y_{a} \leq Y \leq Y_{a}\right\}$ and the condition that $P=0$ on the boundaries. $H$ is given by eqn. (6) and $\epsilon$ is assumed to be a constant. In addition, cavitation is disregarded and the force balance equation plays no role. Discretization of eqn. (9) on a uniform grid with mesh size $h$ gives

$\epsilon\left(P_{i-1, j}+P_{i+1, j}+P_{i, j-1}+P_{i, j+1}-4 P_{i, j}\right) / h^{2}-\left(H_{i, j}-H_{i-1, j}\right) / h=0$

with $H_{i j}$ from eqn. (6). 
The efficiency of a relaxation scheme in reducing high-frequency components can be expressed in terms of its smoothing rate. This is the maximum amplitude amplification factor for those components that can not be represented on the coarser grid. The smoothing rate of a specific relaxation process applied to the solution of eqn. (10) with $H_{i, j}$ given by (6) depends on the ratio $e / h^{2}$. For large values of this ratio eqn. (10) approximates the well known two-dimensional Poisson equation and a simple one-point lexicographic Gauss-Seidel relaxation provides good error smoothing and stability. Unfortunately, with decreasing $\epsilon / h^{2}$ this relaxation gradually becomes unstable. The cause of this instability is that the accumulated changes of the elastic deformation integrals during a relaxation sweep are too large. As a result low-frequency error components are amplified and the scheme becomes unstable.

To find a stable relaxation scheme with good smoothing properties for small $\epsilon$ it is important to ensure that the process remains effectively local, i.e. that relaxing at a point $\left(x_{i}, y_{j}\right)$ introduces only small changes to the discrete integral $I_{k l}$ and in particular to the discrete derivative $\left(H_{k l}-H_{k l-1}\right) / h$ at points $\left(x_{k}, y_{l}\right)$ far away from $\left(x_{i}\right.$, $y_{j}$ ); otherwise each such integral would accumulate too many significant changes in a relaxation sweep. This can be achieved using a suitable kind of distributed relaxation.

In general, a distributed relaxation of the order $r$ is a relaxation where each set of simultaneous changes is an $r$ th-order difference of a local function (e.g. a multiple of a discrete delta-function). Instead of changing the approximation in only one point, changes are also applied at one or more adjacent sites. For example, in the case of a second-order distributive relaxation for a two-dimensional problem five unknowns are changed at a time, i.e. $P_{i j} \leftarrow P_{i j}+\delta_{i j}$ and $P_{i \pm 1, j \pm 1} \leftarrow P_{i \pm 1, j \pm 1-} \delta_{i j} / 4$, where $\delta_{i j}$ is calculated in such a way that, after applying these changes, the equation to be solved, e.g. eqn. (10), is satisfied at $\left(x_{i}, y_{j}\right)$. This relaxation can ensure that the changes in the integrals remain local since the changes in the integral at $\left(x_{k}, y_{l}\right)$ caused by these second-order distributed changes at $\left(x_{i}, y_{j}\right)$ behave like $\partial K^{2}\left(x_{i}, y_{j}, x_{k}, y_{l}\right) / \partial x^{2}+\partial K^{2}$ $\left(x_{i}, y_{j}, x_{k}, y_{l}\right) / \partial y^{2}$, which, in the present case, decays like $\left|\left(x_{i}, y_{j}\right)-\left(x_{k}, y_{l}\right)\right|^{-3}$. Moreover, the changes in the derivative $\mathrm{d} H / \mathrm{d} X$ occurring in the equation will decay even faster.

It can easily be shown that this particular relaxation applied to the present problem is stable, also for the extreme case that $\epsilon=0$. However, there is one complication related to the fact that the problem is two-dimensional. With decreasing $\epsilon$ eqn. (10) reduces to

$\frac{\partial H}{\partial X}=0$

which is a relation in the $X$ direction only. Consequently, when discretized, there is no direct coupling via the pressure between adjacent gridpoints in the $Y$ direction. These points are only indirectly, and very weakly, coupled via the elastic deformation integrals. As a result any relaxation process where the gridpoints are scanned oneby-one, e.g. in lexicographic order, is ineffective in reducing a specific subset of the high-frequency components, $i . e$. those that are smooth with respect to the $X$ direction and high-frequency in the $Y$ direction. For the simple one-point Gauss-Seidel relaxation applied to the full circular contact problem this observation was already made by Lubrecht [8].

Assuming the usual choice of a coarse grid having twice the mesh size of the fine grid with respect to both directions this grid cannot describe those error components and it is essential that they are reduced on the fine grid. This can be achieved rather easily as outlined by Brandt [17], i.e. by using a line relaxation. 
Characteristic for line relaxation is that, instead of scanning all gridpoints one by-one solving changes to satisfy the equation at that point, grid lines are visited one by-one solving all discrete equations of each line simultaneously. These lines should be lines in the direction of the strong coupling, ie. in the present case, lines of constant $Y$ (lines in the $X$ direction).

Summarizing, a good error smoothing process for small $\mathrm{e} / \mathrm{h}^{2}$ must be a distributive line relaxation. For example the following process serves very well in a multilevel solver of the linearized problem: given an approximation $\tilde{P}$ and the associated ap. proximation to the film thickness $\tilde{H}$ all lines of constant $Y(j)$ are visited and it each line simultaneously a new approximation $\tilde{H}_{i, j}$ to $H_{i, j}$ and all changes $\delta_{i, j}(1 \leq i \leq n, \cdots)$ to be applied using the aforementioned distribution are solved from

$$
\begin{aligned}
& \ddot{H}_{i, j}-\frac{2}{\pi^{2}} \sum_{k=1}^{n_{\mathrm{r}}-1} \Delta K_{i k j j}^{\mathrm{hhhh}} \delta_{k, j}=f_{i, j}+\bar{w}_{\mathrm{i}, j} \\
& \epsilon / \mathrm{h}^{2}\left[\left(\tilde{P}_{i-1, j}-\delta_{i-2, j} / 4+\delta_{i-1, j}-\delta_{i, j} / 4\right)+\left(\tilde{P}_{i+1, j}-\delta_{i, j} / 4+\delta_{i+1, j}-\delta_{i+2, j} / 4\right)\right. \\
& \left.\quad-4\left(\tilde{P}_{i, j}-\delta_{i-1, j} / 4+\delta_{i, j}-\delta_{i+1, j} / 4\right)+\left(\tilde{P}_{i, j+1}-\delta_{i, j} / 4\right)+\left(\tilde{P}_{i, j-1}-\delta_{i, j} / 4\right)\right] \\
& \quad-\left(\bar{H}_{i, j}-\bar{H}_{i-1, j}\right) / h=0
\end{aligned}
$$

where

$\Delta K_{i i j j}^{\text {hhhh }}=K_{i i j j}^{h h h h}-\left(K_{i i-1 j j}^{h h h h}+K_{i i+1 j j}^{h h h h}+K_{i i j i-1}^{h i h h}+K_{i i j j+1}^{j i h h h_{i}}\right) / 4$

In eqn. (12) $\bar{w}_{i j}$ denotes the elastic deformation summation

$\tilde{w}_{i j}=\frac{2}{\pi^{2}} \sum_{k=0}^{n_{x}} \sum_{l=0}^{n_{y}} K_{i k j l}^{A h h l} \ddot{P}_{k l}$

whereas $f_{i j}$ stands for

$f_{i j}=H_{\infty 0}+\frac{X_{i}^{2}}{2}+\frac{Y_{j}^{2}}{2}$

After all interior lines $j$ have been visited the changes are applied according to

$\tilde{P}_{i, j}=\tilde{P}_{i, j}+\delta_{i, j}-\left(\delta_{i-1, j}+\delta_{i+1, j}+\delta_{i, j-1}+\delta_{i, j+1}\right) / 4$

and the new values of the pressure $\bar{P}_{i, j}$ are used to update or recalculate the approximation to the elastic deformation $\left(w_{i j}^{h}\right)$. This Jacobi distributive line relaxation scheme is very effective in reducing high-frequency error components. The only complication is how to solve all changes together with the new values of $H_{i,}$, on one line simultaneously. In fact, this requires the solution of a (full) system of $O\left(n^{1 / 2}\right)$ discrete equations, where $n$ is the total number of nodes on the grid. However, to obtain the full line relaxation efficiency, the equations need not be solved exactly. In general it is sufficient if the error on that line is reduced by say one or two orders of magnitude. Therefore, since $\Delta K_{i k j i}^{\text {hhhh }}$ decreases very fast with increasing distance $|i-k|$, it is sufficient to take into account only the threc largest terms of the summation and to solve a reduced system of equations.

There are various alternatives enabling a fast solution of all $\bar{H}_{i, j}$ and $\delta_{i, j}$ from the reduced system. An effective way is for examplc by mcans of decimation. This process and its implementation for the linear model problem considered here are explained in detail in ref. 14 (appendix D).

The line relaxation process described above was implemented in a multilevel solver for the model problem and for small $\epsilon / h^{2}$, particularly for the extreme case $\epsilon=0$, an 
error reduction of an order of magnitude per coarse grid correction cycle $(V(2,1)$ or $W(2,1)$ see ref. 16) was easily obtained.

Also for larger values of $\epsilon / h^{2}$ the relaxation scheme still rather effectively reduces high-frequency error components and even in the limiting case of very large $\epsilon / h^{2}$, i.e. the two-dimensional Poisson problem, the asymptotic smoothing rate $\bar{\mu}$ is still 0.6 . However, for these large values of $\epsilon / h^{2}$ the present relaxation process is outranked by the simple one-point Gauss-Seidel relaxation with lexicographic ordering which has an asymptotic smoothing rate of 0.5 . An even better alternative for large values of $\epsilon / h^{2}$ is a Gauss-Seidel line relaxation with an asymptotic smoothing rate of 0.4 . This latter relaxation can be described as follows: For each line of constant $Y(j)$ changes $\delta_{i j}$ and a new approximation $\tilde{H}_{i, j}$ to $H_{i, j}$ are solved simultaneously from

$$
\begin{aligned}
& \ddot{H}_{i, j}-\frac{2}{\pi^{2}} \sum_{k-1}^{n_{x}-1} K_{i k, j j} \delta_{k, j}=f_{i, j}+\bar{w}_{i, j} \\
& \epsilon / h^{2}\left[\left(\tilde{P}_{i-1, j}+\delta_{i-1, j}\right)+\left(\tilde{P}_{i+1, j}+\delta_{i+1, j}\right)-4\left(\tilde{P}_{i, j}+\delta_{i, j}\right)+\bar{P}_{i, j+1}+\bar{P}_{i, j-1}\right] \\
& \quad-\left(\bar{H}_{i, j}-\bar{H}_{i-1, j}\right) / h=0
\end{aligned}
$$

When all $\delta_{i, j}$ for a line $j$ are solved they are applied immediately

$\tilde{P}_{i, j}=\tilde{P}_{i, j}+\hat{\delta}_{i, j}$

These new values of $P$ on line $j$ are subsequently used when treating the next line as is characteristic for a successive displacement scheme. In order to obtain the full efficiency it is not necessary to take into account all terms of the summation. Since $K_{i k j i}^{\text {hhh }}$ decreases with distance as $|i-k|^{-1}$ it is, as in the procedure described above, sufficient to take into account only three terms.

As was found for the simple (one-point) Gauss-Seidel relaxation the Gauss-Seidel line relaxation, although it does not suffer from the loss of coupling, is not stable for small values of $\epsilon / h^{2}$. Below $\epsilon / h^{2} \approx 0.3$ low-frequency crror components arc amplified and the process diverges. Therefore, a multilevel solver for the model problem giving good results for all $\epsilon$ was obtained by combining two relaxations, i.e.:

on grids where $\epsilon / h^{2} \geq 0.3$ the Gauss-Seidel line relaxation is used.

on grids where $\epsilon / h^{2}<0.3$ the Jacobi distributive line relaxation is used.

In this way an error reduction of one order of magnitude per coarse grid correction cycle $(V(2,1)$ or $W(2,1))$ was obtained regardless of the value of $\epsilon$.

\subsubsection{Varying coefficients}

The next step leading to a solver for the circular contact problem is to consider eqn. (1) and allow the coefficient $\epsilon$ to vary over the grid, i.e. to solve the problem

$\frac{\partial}{\partial X}\left(\epsilon \frac{\partial P}{\partial X}\right)+\frac{\partial}{\partial Y}\left(\epsilon \frac{\partial P}{\partial Y}\right)-\frac{\partial H}{\partial X}=0$

on the domain $\left\{(X, Y) \in \mathbb{R}^{2} \mid X_{a} \leq X \leq X_{b},-Y_{a} \leq Y \leq Y_{a}\right\}$ with the condition that $P=0$ on the boundaries and $H$ is given by eqn. (6) for different functions $\epsilon(X, Y)$. Cavitation is disregarded and the force balance equation plays no role. To approximate the full circular contact problem $\epsilon(X, Y)$ is chosen as

$\epsilon(X, Y)= \begin{cases}\left(X^{2}+Y^{2}\right)^{3} / 8 & \text { if } X^{2}+Y^{2}>1 \\ 0 & \text { otherwise }\end{cases}$

Equation (19) is discretized according to 


$$
\begin{aligned}
& h^{-2}\left(\epsilon_{i-1 / 2, j}\left(P_{i-1, j}-P_{i, j}\right)+\epsilon_{i+1 / 2, j}\left(P_{i+1, j}-P_{i, j}\right)+\epsilon_{i, j-1 / 2}\left(P_{i, j-1}-P_{i, j}\right)\right. \\
& \left.\quad+\epsilon_{i, j+1 / 2}\left(P_{i, j+1}-P_{i, j}\right)\right)-h^{-1}\left(\bar{\rho}_{i} H_{i}-\bar{\rho}_{i, 1} H_{i-1}\right)=0
\end{aligned}
$$

$\epsilon_{i-1 / 2, j}, \epsilon_{i+1 / 2, j}, \epsilon_{i, j-1 / 2}$ and $\epsilon_{i, j+1 / 2}$ denote the value of $\epsilon(X, Y)$ at the intermediat locations as described before.

In the multilevel algorithm described in section 3.3.1 the relaxation type used on a specific grid depends on the value of $\epsilon / h^{2}$. As mentioned before, relaxation is, by its nature, a local process. Hence different relaxations can be combined on one grid. For the specific problem considered here the two-line relaxation processes can be combined in the following way: for a given line of constant $Y$ the changes $\delta_{i, j}$ and the new approximations to $\bar{H}_{i, j}$ are solved from a system of equations, i.e. two equations per gridpoint $i$. If at least one of $\epsilon_{i \pm 1 / 2, j \pm 1 / 2} / h^{2}$ exceeds the value of the crossover point the two equations for this grid point are

$$
\begin{aligned}
& \bar{H}_{i, j}-\frac{2}{\pi^{2}} \sum_{k=1}^{n_{x}-1} K_{i k, j j} \delta_{k, j}=f_{i, j}+\tilde{w}_{i, j} \\
& h^{-2}\left[\left(\epsilon_{i-1 / 2, j}\left(\tilde{P}_{i-1, j}+\delta_{i-1, j}\right)+\epsilon_{i+1 / 2, j}\left(\tilde{P}_{i+1, j}+\delta_{i+1, j}\right)\right.\right. \\
& \left.\quad-\left(\epsilon_{i, j-1 / 2}+\epsilon_{i, j+1 / 2}+\epsilon_{i+1 / 2, j}+\epsilon_{i-1 / 2, j}\right)\left(\tilde{P}_{i, j}+\delta_{i, j}\right)+\epsilon_{i, j+1 / 2} \tilde{P}_{i, j+1}+\epsilon_{i, j-1 / 2} \ddot{P}_{i, j-1}\right] \\
& \quad-\left(\bar{H}_{i, j}-\bar{H}_{i-1, j}\right) / h=0
\end{aligned}
$$

Otherwise, i.e. if all $\epsilon_{i \pm 1 / 2, j \pm 1 / 2} / h^{2}$ are smaller than the value of the crossover point, the two equations for this grid point are

$$
\begin{aligned}
\bar{H}_{i, j} & -\frac{2}{\pi^{2}} \sum_{k=1}^{n_{x}-1} \Delta K_{i k j j}^{h h h h} \delta_{k, j}=f_{i, j}+\tilde{w}_{i, j} \\
h^{-2} & {\left[\epsilon_{i-1 / 2, j}\left(\tilde{P}_{i-1, j}-\delta_{i-2, j} / 4+\delta_{i-1, j}-\delta_{i, j} / 4\right)\right.} \\
& +\epsilon_{i+1 / 2, j}\left(\tilde{P}_{i+1, j}-\delta_{i, j} / 4+\delta_{i+1, j}-\delta_{i+2, j} / 4\right) \\
& +\epsilon_{i, j+1 / 2}\left(\tilde{P}_{i, j+1}-\delta_{i, j} / 4\right)+\epsilon_{i, j-1 / 2}\left(\bar{P}_{i, j-1}-\delta_{i, j} / 4\right) \\
& \left.-\left(\epsilon_{i, j-1 / 2}+\epsilon_{i, j+1 / 2}+\epsilon_{i+1 / 2, j}+\epsilon_{i-1 / 2, j}\right)+\left(\tilde{P}_{i, j}-\delta_{i-1, j} / 4+\delta_{i, j}-\delta_{i+1, j} / 4\right)\right] \\
& -\left(\bar{H}_{i, j}-\bar{H}_{i-1, j}\right) / h=0
\end{aligned}
$$

In both situations it is sufficient to solve a reduced version of the system of equations, i.e. see ref. 14, appendix D. Once all $\delta_{i, j}$ and $\bar{H}_{i, j}$ for the specific line are solved $\delta_{i, j}$ is added to $\tilde{P}_{i, j}$ or, when the sweep is completed, to $\tilde{P}_{i, j}$ and partly to its four neighbours depending on the equations solved for the gridpoint $(i, j)$.

Using this hybrid relaxation scheme in a multilevel solver for eqn. (21) with $\epsilon(X$, $Y$ ) given by (20), a reduction of the error by almost one order of magnitude (a factor of 8 ) per coarse grid correction cycle was obtained.

\subsubsection{Circular contact}

In the previous section the coefficient $\epsilon$ was given as a function of the spatial coordinates $X$ and $Y$. The ncxt stcp towards a relaxation scheme for the full nonlinear circular contact problem is to give $\epsilon$ as a function of $H$ and $P$ instead. This can be done straightforwardly since the description of the relaxation scheme in the previous section was entirely given in terms of $\epsilon$, i.e. instcad of using eqn. (20), $\epsilon_{i, j}$ is calculated according to: 
$\epsilon_{i, j}=\frac{\bar{\rho}\left(\tilde{P}_{i, j}\right) \bar{H}_{i, j}^{3}}{\bar{\eta}\left(\bar{P}_{i, j}\right) \lambda}$

In addition, $\partial H / \partial X$ is replaced by $\partial(\bar{\rho} H) / \partial X$ and when applying the pressure changes the cavitation condition is imposed, i.e. negative values of $P$ are not allowed. The last step is to add the force balance equation to the system. Once every $s$ relaxations the integration constant $H_{00}$ is adjusted according to:

$H_{00} \longleftarrow H_{00}-c\left(\frac{2 \pi}{3}-h^{2} \sum_{i=1}^{n_{x}-1} \sum_{j=1}^{n_{y}-1} \tilde{P}_{i, j}\right)$

where $\vec{P}_{i, j}$ is the current approximation to the pressure profile. The number of relaxations after which the force balance equation is relaxed, $s$, must be large enough and the multiplication factor $c$ must be sufficiently small to avoid unstable oscillations. On the other hand, $c$ and $s$ should also be chosen in such a way that the overall convergence is not slowed down too much. The reader is reminded that in the case where multiple grids are used the force balance equation is only relaxed on the coarsest grid, i.e. see ref. 8 and also ref. 15 .

This concludes the description of a relaxation scheme that provides a stable solver for the circular contact problem. Because of the nonlinearity, underrelaxation is needed. The necessary underrelaxation depends on the load conditions and, as experience has shown, varies from 0.3 to 1.0 for the distributed changes and from 0.6 to 1.0 for the single changes. In addition, in particular for high loads, the mesh size on the grid should be sufficiently small.

\section{Example}

To check convergence of the solution of the continuous integro-differential problem and to check the complexity of the algorithm the results obtained for a specific load situation are studied in detail. The values of the Moes dimensionless point contact parameters (see nomenclature) for this load situation are: $M=50$ and $L=10$. This coincides with $\bar{\alpha}=13.4$ and $\lambda=0.06$. Upon assuming $\alpha=1.7 \times 10^{-8}$ the maximum Hertzian pressure for this load situation is $0.8 \mathrm{GPa}$. Hence, it is a relatively lightly loaded situation, but in the present paper it serves well for demonstration purposes. Values of the Hamrock and Dowson dimensionless point contact parameters describing the same load situation are: $W_{h d}=4.73 \times 10^{-7}, U=1.0 \times 10^{-11}$, and $G=4728$.

\subsection{Calculational details}

The solution has been calculated on a uniform grid covering the domain $\{(X$, $\left.Y) \in \mathbb{R}^{2} \mid-4.5 \leq X \leq 1.5,-3 \leq Y \leq 3\right\}$. A Full Multigrid (FMG) algorithm $[8,15]$ was used with two coarse grid correction cycles $(W(2,1))$ per refinement, each cycle giving an error reduction of almost one order of magnitude. The coarsest grid used in the FMG algorithm, denoted by level 1 , consisted of $(8+1) \times(8+1)$ nodes and the finest grid consisted of $(512+1) \times(512+1)$ nodes. Hence, the solution has been calculated using as much as 263,169 nodal points.

\subsection{Solution}

Figures 1 and 2 show the calculated pressure profile and the associated film shape. It may be obvious that, with the number of nodes used in the present calculations, the usual presentation of the results in a "wire" figure, is impossible (it becomes 


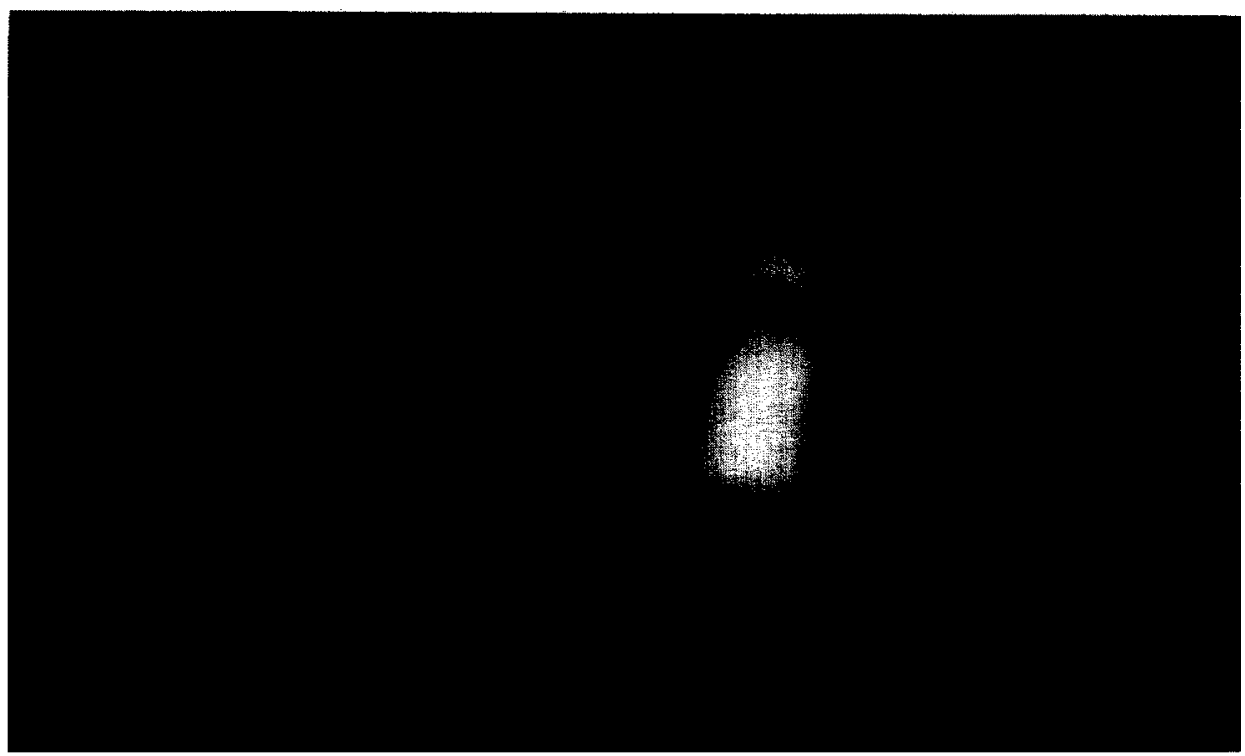

Fig. 1. Dimensionless pressure profile $(P)$ circular contact, $M=50, L=10$.

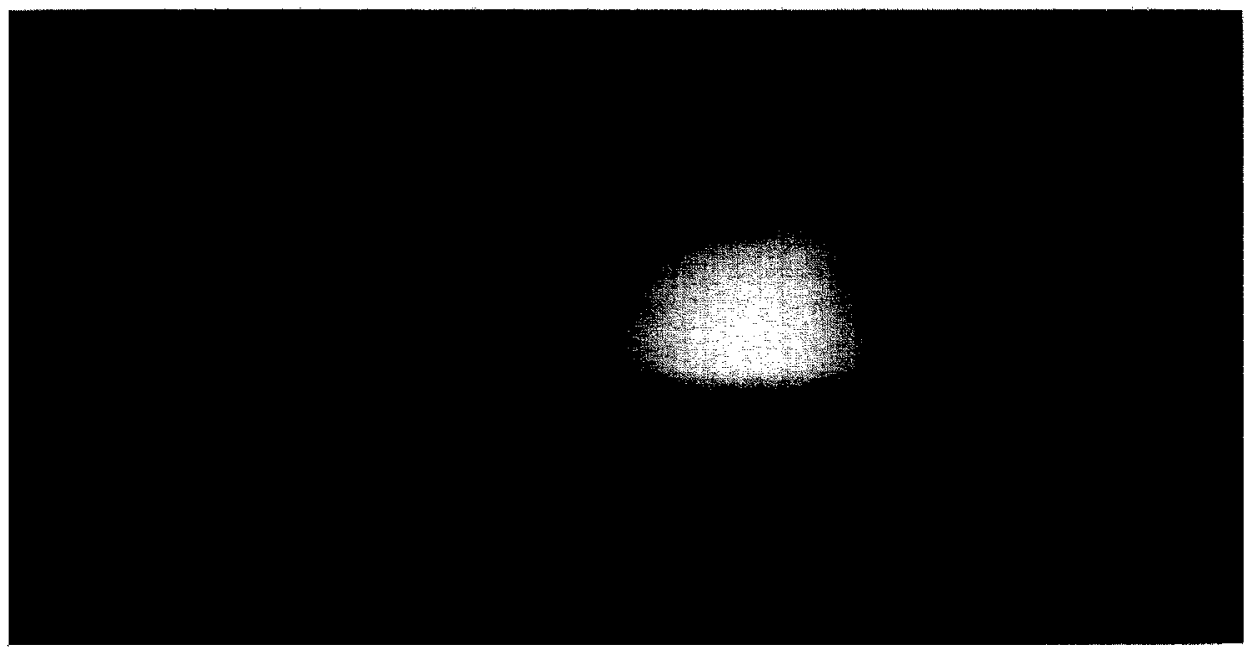

Fig. 2. Dimensionless film shape $(H)$ circular contact, $M=50, L=10$.

totally black); therefore, the results are presented using colorgraphics. The different shades represent different angles between the outward normal on the profile and the viewer.

These figures show all characteristic elements of medium to highly loaded EHL circular contact solutions. Firstly, the pressure profile resembles the Hertzian dry contact semi-ellipsoid pressure profile. In the inlet region the pressure gradually builds up to the semi-ellipsoid. The cavitated region is preceded by the three-dimensional version of the pressure spike. Note that, compared with the one-dimensional situation, 
this "spike" is a shield of high-pressure values "wrapped around" the Hertzian contact region. Secondly, the film thickness graph clearly displays the formation of the socalled side-lobes or of a horseshoe-shaped region. The overall minimum film thickness occurs at these side lobes and its value deviates significantly from the minimum film thickness found on the centerline of the contact.

An alternative way of presenting the calculational results is by means of contour line plots, i.e. drawing lines along which the film thickness or the pressure is constant. Such graphs for the pressure and the film thickness are presented in Figs. 3 and 4. In Fig. 3 the pressure spike region can be recognized easily. It is the dark region of large gradients. Also the fact that the spike is in fact a shield wrapped around a more or less semi-ellipsoid is clearly visualised. The film thickness contour graph (Fig. 4) clearly displays the horseshoe-shaped region, i.e. the side lobes. Note that the region of large gradients in the film thickness in Fig. 4 coincides with the region of large gradients in the pressure in Fig. 3.

\subsection{Convergence}

As has been explained by Lubrecht [8] the use of a FMG algorithm allows an easy check whether the calculated solution has converged below the level of the discretization error. In addition, the order of convergence of the solution of the discrete problem to the solution of the continuous integro-differential problem with decreasing mesh size can be checked. This latter check can be done by monitoring the value of a characteristic result such as the minimum or central film thickness as a function of the mesh size on the grid.

For example, Table 1 gives the value of the minimum and central film thickness as a function of the mesh size for the load situation considered here. Table 1 also displays the ratio between the minimum and central film thickness. From a sufficiently small mesh size onwards, with decreasing mesh size a first-order convergence to a limiting value can be observed. This is exactly what could be expected hecause of the
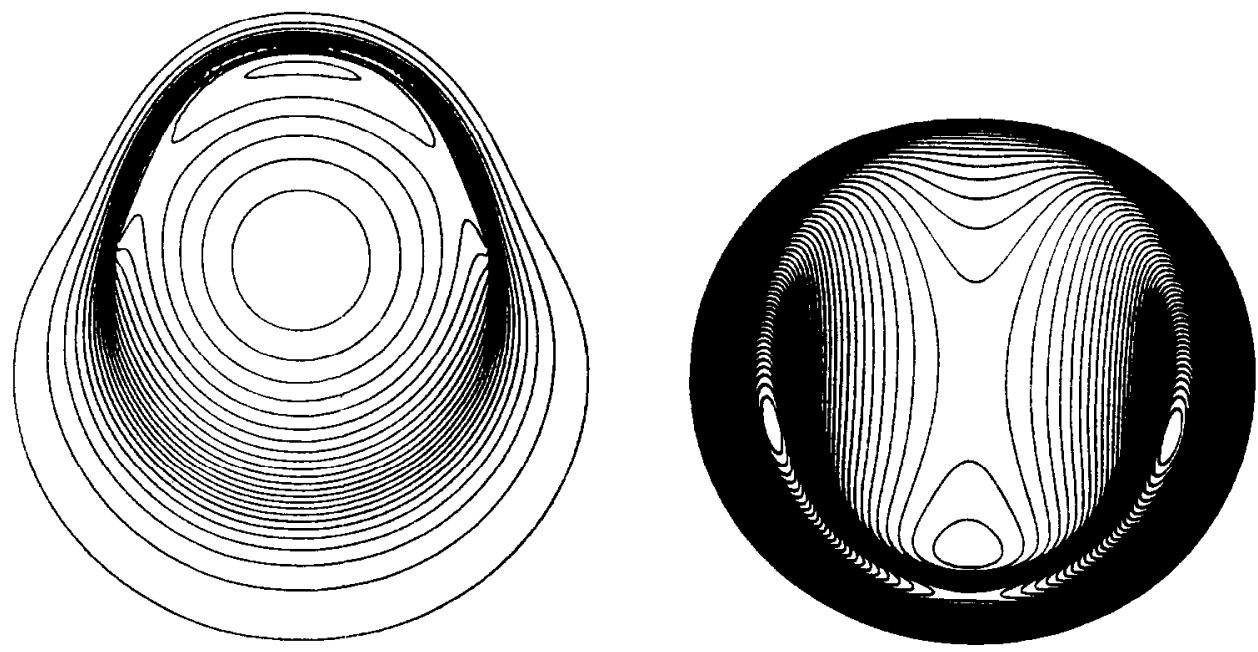

Fig. 3. Contour line plot of the pressure profile displayed in Fig. 1, $(M=50, L=10) \Delta P=0.04$.

Fig. 4. Contour line plot of the film shape displayed in Fig. 1, $(M=50, L=10) \Delta H=0.0025$. 
TABLE 1

Minimum and central film thickness as a function of the number of nodes (mesh size;

\begin{tabular}{|c|c|c|c|c|c|}
\hline Level & $n_{x}+1$ & $n_{y}+1$ & $H_{1 \mathrm{n}}$ & $H_{i}$ & $H H_{n}$ \\
\hline 2 & 17 & 17 & $8.868 \times 10^{2}$ & $6.875 \times 10^{-1}$ & 43 \\
\hline 3 & 33 & 33 & $1.525 \times 10^{-1}$ & $1.967 \times 10^{\circ-1}$ & 200 \\
\hline 4 & 65 & 65 & $1.668 \times 10^{1}$ & $2.667 \times 10^{\circ}$ & $\therefore 509$ \\
\hline 5 & 129 & 129 & $1.650 \times 10^{-1}$ & $2686 \times 10^{-1}$ & 0,30 \\
\hline 6 & 257 & 257 & $1.624 \times 10^{-5}$ & $2.649 \times 10^{-1}$ & 836 \\
\hline 7 & 513 & 513 & $1.609 \times 10^{-3}$ & $2.620 \times 10^{-1}$ & $: 639$ \\
\hline
\end{tabular}

TABLE 2

Computing time on a HP $900 / 840$ s computer as a function of the number of nodes for the FMG algorithm with two $V(2,1)$ cycles and two $W(2,1)$ cycles

\begin{tabular}{ccccc}
\hline$k$ & $n_{x}+1$ & $n_{y}+1$ & $2 V(2,1)$ & $2 W(2,1)$ \\
\hline 2 & 17 & 17 & 9.5 & 9.5 \\
3 & 33 & 33 & 22.1 & 40.3 \\
4 & 65 & 65 & $1: 23$ & $2: 44$ \\
5 & 129 & 120 & $4: 51$ & $10: 40$ \\
6 & 257 & 257 & 17.35 & 4127 \\
7 & 513 & 513 & 1.07 .53 & $2: 41.25$ \\
\hline
\end{tabular}

first-order upstream discretization of the wedge term in Reynolds' equation and similar results were obtained for the line contact problem, see ref. 15 . Only in very lightly loaded situations where the wedge term is small compared with the Poiseulle terms second-order convergence can be observed, see also ref. 7 .

\subsection{Computing times}

To conclude this example the computing time required to obtain a solution is studied. Table 2 presents computing times used by the FMG algorithm with two $V(2$, 1) and two $W(2,1)$ cycles per grid refinement as a function of the number of nodes. The first column is representative for lightly loaded situations whereas the second column is representative for moderate to highly loaded situations. The data displayed in Table 2 are also shown in Fig. 5. For reasons of comparison, in this figure a line representing the computing time of $O(n)$ and a line representing the computing time of $O\left(n^{2}\right)$ are also drawn. Obviously the complexity of the algorithm is very close to $O(n)$. Halving the mesh sizes, i.e. increasing the number of nodes by a factor of 4 , the computing time increases by a factor of 4 . The fact that it is actually $O(n \ln n)$ cannot even be recognized.

\section{Conclusions}

An alternative relaxation process for the EHL circular contact problem was developed. Using common multigrid techniques to accelerate convergence of this relaxation process together with the recently developed multilevel technique Multilevel 


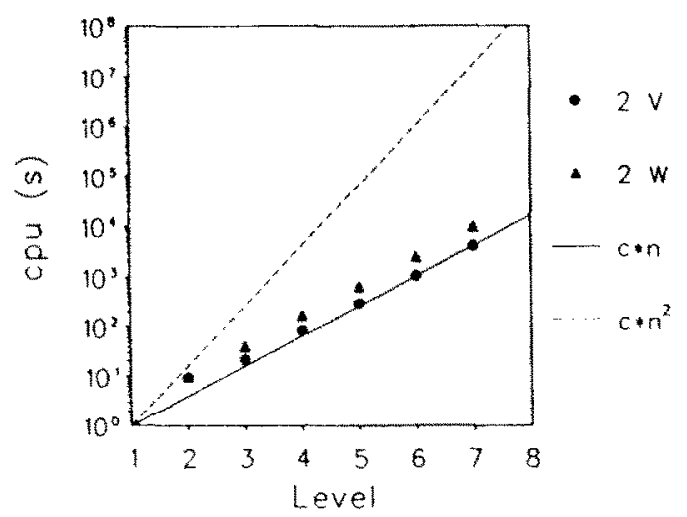

Fig. 5. Computing time on a HP $9000 / 845$ s computer for the $\mathrm{FMG}$ algorithm with two $V(2,1)$ cycles and two $W(2,1)$ cycles.

Multi-integration for the fast evaluation of the elastic deformation resulted in an algorithm solving the EHL circular contact problem in $O(n \ln n)$ operations. This low complexity was demonstrated by means of an example. It was shown that, as a result, the algorithm allows solution of the problem with a large number of nodes, e.g. 250000 on a minicomputer. This first part focused on a description of the numerical algorithm. The second part presents the results obtained with this solver for the "standard" circular contact problem, i.e. assuming the surfaces to be ideally smooth. The results presented in that part show that the algorithm is not only fast but also very stable.

Although the present paper is restricted to a circular contact situation, the very same algorithm outlined here can be used in the study of elliptical contact situations.

\section{Acknowledgments}

The authors would like to thank Professor A. Brandt of the Weizmann Institute of Science, Rehovot, Israel for many helpful suggestions and discussions on the development of the relaxation scheme. They would also like to thank Dr. A. A. Lubrecht of the SKF Engineering and Research Centre B.V., Nieuwegein, The Netherlands, for his help with respect to the Multilevel Multi-Integration techniques.

Part of the work presented here was done during a visit to Professor Brandt at the Weizmann Institute of Science, Rehovot, Israel sponsored by the Dutch foundations KIVI and NWO and during a visit to Professor Brandt at the University of Colorado, Denver, U.S.A. sponsored by the faculty of Mechanical Engineering of the University of Twente. The research was also sponsored by SKF Engineering and Research Centre B.V., Nieuwegein, The Netherlands.

The photographs presented in this paper were made by TTF, Enschede, The Netherlands.

\section{References}

1 R. J. Chittenden, D. Dowson, J. F. Dunn and C. M. Taylor, A theoretical analysis of the isothermal elastohydrodynamic lubrication of concentrated contacts I. Direction of lubricant entrainment coincident with the major axis of the Hertzian contact ellipse, Proc. $R$. Soc. London A, 397 (1985) 245-269. 
2 R. J. Chittenden, D. Dowson, J. F, Dunn and C. M. Tayor, A theoretical analys if in isothermal elastohydrodynamic lubrication of concentrated contacts II. General case, with lubricant entrainment along either principal axis of the Hertzian contact cllipse an an intermediate angle, Proc $R$ So Lomdon A, 397 (1985:271-204

3 B. J. Hamrock and D. Dowson, Isothernal elastohydrodynamic lubrication of nom antics part 1 - Theoretical Formulation, ASME JOT, $98(1976) 223229$.

4 D. Zhu and H. S. Cheng. Fffect of surtace roughness on the point contact UH s smt JOT, $110(1988) 32,37$.

$5 \mathrm{~K} . \mathrm{P}$. Oh, The numerical solution of dynamically loated elastohydrodynamic annat as a nonlinear complementarity problem, ASME JOT, 100 (1985) 88.95 .

$6 \mathrm{~K}$. P. Oh and S. M. Rohde, Numerical solution of the point contact EHL problem using the Finite Flement Method, Imt I. Nimer. Meth. in Frit. // (1977) 1507-1518.

7 A. A. Lubfecht, Multigrid, an alternative method of solution for two-dimensional clasto. hydrodynamically lubricated point contact calculations. ASME JOT, 109 (1987) 437.43

8 A. A. Lubrecht, The numerical solution of the clastohydrodynamically lubricated ima- and point contact problem using multigrid techniques, Ph.D. Thesis Univ of Twente, Ensehede. 1987; ISBN 90-9001583-3.

9 C. C. Kweh, H. P. Evans and R. W. Snidie, Elastohydrodyname lubrication of heavily inded circular contacts, Proc. Inst. Mech. Eng., 203 (1989) 133-148.

$10 \mathrm{~J}$. Seabra and D. Berthe, Elastohydrodynamic point contacts part 1: Formulation and numerical solution, Wear, 130 (1989) 301-318.

11 A. Brandt and A. A. Lubrecht, Multilevet matrix multiplication and fast solution of integrat equations, J. Comput. Phys, 90 (1990) 348-370.

12 D. Dowson and G. R. Higginson, Elasto-hydrodynamic lubrication. The fundamentals of roller and gear lubrication, Pergamon, Oxford, 196\%.

13 C. J. A. Roelands, Correlational aspects of the viscosity-temperature-pressure rclotionship of lubricating oils, Ph.D. Thesis, Technische Hogeschool Delft. The Netherlands, 1966 (V.R.B., Groningen, The Netherlands)

$14 \mathrm{C} . \mathrm{H}$. Venner, Multilevel solution of the EHL line and point contact problems, Ph.D Thesis. Univ. of Twente, Enschede, The Netherlands, 1991; ISBN 90-9003974-01.

15 C. H. Venner, W. E. ten Napel and R. Bosma, Advanced multilevel sslution of the LHI. line contact problem, ASME JOT, $112(1090) 426-432$

16 W. L. Briggs, A Multigrid Tutorial, SIAM, Philadelphia, PA, 1987, ISBN 0-89871-221-1.

17 A. Brandt, Multigrid Techniques: 1984 Guide with applications to fluid dyntamics, available as G. M. D.-Studien No. 85, from G.M.D.F1T, Postfach 1240, D-5205, St Augustin 1. F.R.G. 1984.

18 A. A. Lubrecht and E. Ioannides, $A$ fast solution of the dry contact problem and the associated sub-surface stress field, using multilevel techniques, ASME JOT, 113 (1991) 128 -133.

\section{Nomenclature}

$\begin{array}{ll}a & \left.\text { half-width Hertzian contact region, } a=\left(\left(3 w R_{x}\right) / 2 E^{\prime}\right)\right)^{1 / 3} \\ E & \text { elasticity modulus (Young's modulus }) \\ E^{\prime} & \text { reduced modulus of elasticity, } 2 / E^{\prime}=\left(1-\nu_{1}^{2}\right) / E_{1}+\left(1-\nu_{2}^{2}\right) / E_{2} \\ G & \text { material parameter, } G=\alpha E^{\prime} \\ h & \text { film thickness } \\ H & \text { dimensionless film thickness, } H=(h R) / a^{z} \\ H_{00} & \text { integration constant in dimensionless film thickness equation } \\ K_{i k h h t}^{\text {thh }} & \text { discretized kerncl in film thickness equation } \\ L & \text { dimensionless material parameter (Moes), } L=G(2 U)^{1 / 4} \\ M & \text { dimensionless load parameter (Moes), } M=W(2 U)^{-3 / 4} \\ p & \text { pressure } \\ p_{\mathrm{h}} & \text { maximum Hertzian pressure, } p_{\mathrm{h}}=(3 F) /\left(2 \pi a^{2}\right)\end{array}$




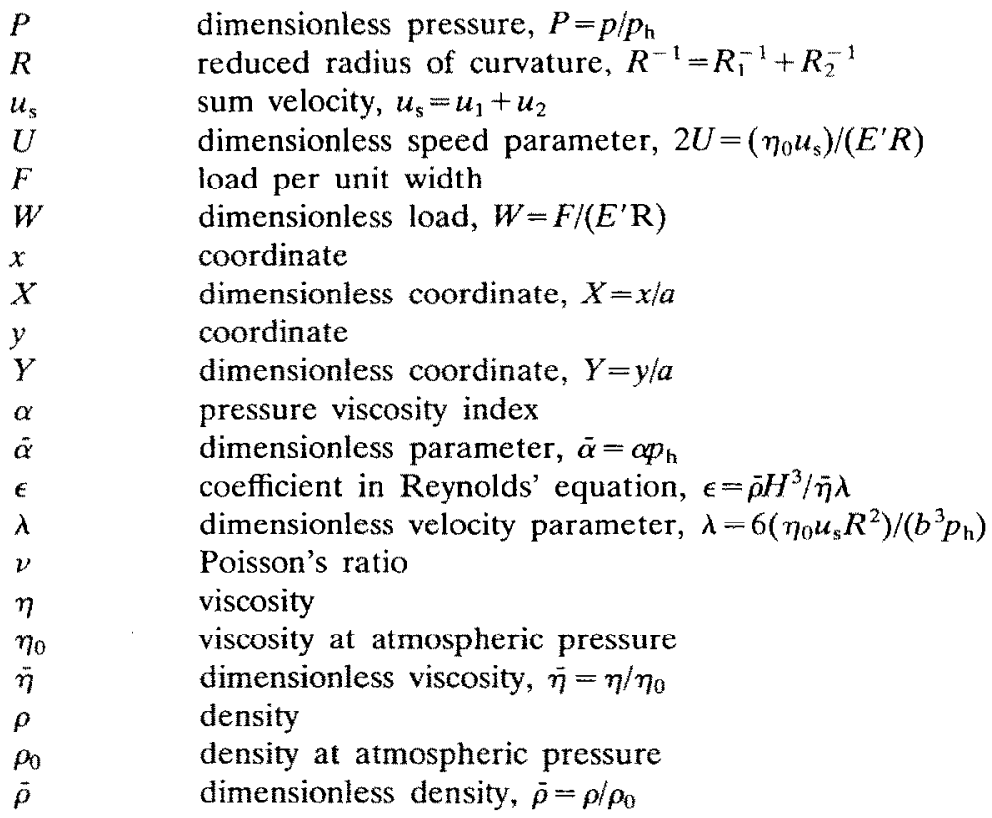

\section{Sub-, superscripts}

$\begin{array}{ll}1,2 & \text { body } 1,2 \\ x, y & x, y \text { direction } \\ \mathrm{m} & \text { minimum } \\ \mathrm{c} & \text { central } \\ i, j & \text { grid index } \\ k, l & \text { grid index }\end{array}$

